\title{
A Frequency Tuning Method for a Planar Inverted-F Antenna
}

\author{
Mikko Komulainen, Student Member, IEEE, Markus Berg, Student Member, IEEE, Heli Jantunen, Member, IEEE, \\ Erkki T. Salonen, and Charles Free, Member, IEEE
}

\begin{abstract}
A novel method is presented for electrically tuning the frequency of a planar inverted-F antenna (PIFA). A tuning circuit, comprising an RF switch and discrete passive components, has been completely integrated into the antenna element, which is thus free of $\mathrm{dc}$ wires. The proposed tuning method has been demonstrated with a dual-band PIFA capable of operating in four frequency bands. The antenna covers the GSM850, GSM900, GSM1800, PCS1900 and UMTS frequency ranges with over $40 \%$ total efficiency. The impact of the tuning circuit on the antenna's efficiency and radiation pattern have been experimentally studied through comparison with the performance of a reference antenna not incorporating the tuning circuit. The proposed frequency tuning concept can be extended to more complex PIFA structures as well as other types of antennas to give enhanced electrical performance.
\end{abstract}

Index Terms-Frequency tuning, mobile antennas, planar inverted-F antenna (PIFA), RF switches.

\section{INTRODUCTION}

$\mathbf{T}$ HE ever-increasing number of wireless communication standards being applied to handheld devices has created a need for integrated antennas operating in ten or more distinct frequency bands [1]. Also, there is a trend toward highly integrated devices that are slimmer, smaller, and lighter than those in current use. However, this creates problem for the antenna designer, since he is limited by fundamental relationships between antenna size, bandwidth, and efficiency [2]. Thus, covering a single very wide frequency band, or multiple frequency bands, whilst maintaining small size and high efficiency is major challenge to the designer. To some extent the problem of covering wide bandwidths can be overcome through the use of multiple antennas, but then mutual coupling between the individual antennas may cause severe problems.

Recent studies of handset antennas have mainly focused on small, wideband or multiband antenna solutions. However, an-

Manuscript received April 26, 2007; revised November 7, 2007. This work was supported in part by the Finnish Funding Agency for Technology and Innovation (project number 52292), in part by the Finnish Society of Electrical Engineers, the Ulla Tuominen Foundation, the Tauno Tönning Foundation, the Nokia Foundation, the Seppo Säynäjäkangas Foundation, the Jenny and Antti Wihuri Foundation, the University of Oulu Foundation, and in part by the Riitta and Jorma J. Takanen Foundation.

M. Komulainen and H. Jantunen are with the Microelectronics and Materials Physics Laboratories and the Electronics Materials, Packaging and Reliability Techniques (EMPART) Research Group of Infotech Oulu, University of Oulu, FIN-90014 Oulu, Finland (e-mail: mikko.komulainen@ee.oulu.fi).

M. Berg and E. T. Salonen are with the Telecommunication Laboratory, University of Oulu, FIN-90014 Oulu, Finland.

C. Free is with the Advanced Technology Institute, University of Surrey, Guildford, Surrey GU2 7XH, U.K.

Digital Object Identifier 10.1109/TAP.2008.919200 tennas that have a reconfigurable operating frequency together with frequency-invariant radiation characteristics will have clear technical benefits. Whilst this kind of antenna will not cover all the frequency bands simultaneously, it may provide several dynamically selectable narrow frequency bands and, within these bands, exhibit higher efficiency than is achievable with conventional antenna solutions [3]. This approach may be used to downsize the antennas whilst maintaining the operating bandwidth or, alternatively, to increase the bandwidth of the antenna without increasing the physical size. In addition, the antenna tuning can be used to compensate for proximity effects that occur when the operating frequencies of handset antennas are detuned by the proximity of the user or by changes in the operating environment, such as occur when opening and closing a clamshell or a swivel- type mobile phone [1], [4]. In a wider context, frequency-tunable antennas are used in space and military applications and are proposed as one of the key enablers for the futures software defined radios [3], [5]-[7]. However, there are some conceivable drawbacks to frequency tunable antennas, which exhibit high RF power loss, limited power handling capability, poor linearity, and especially high dc power consumption of active tuning circuit components. It should also be noted that these antennas are often complicated structures to design and implement, which may have cost and reliability implications. Withstanding these limitations, frequency tunable antennas provide significant technical and economic advantages over their conventional counterparts.

Planar inverted-F antennas (PIFAs) are among the most widely used antennas in mobile terminals. PIFAs can be modified to operate over multiple frequency bands by using, for example, parasitic antenna elements and/or slitting the antenna element to form several paths for surface currents. Recently, PIFA structures covering the frequency range of six telecommunication standards have been reported [8], [9]. A common approach to tuning the operating frequency of a PIFA is to electrically reconfigure a short circuit connection with an external tuning circuit. This has been used for single-band and dual-band antennas [10]-[13]. Other previously reported tuning methods include the use of an adjustable reactive component between a PIFA patch and a ground plane [10], [14], [15], or switched tuning stubs, which have been applied in both single-band and dual-band PIFAs [16], [17]. In these tuning methods, the tuning circuit is separated from the antenna and built either next to, or under, the antenna element. Tunable antennas implemented in this way utilize separate dc wiring for controlling the state of the RF switches or variable capacitors. These wires, like any metal objects located in the vicinity of an antenna, may cause severe performance degradation, such as 


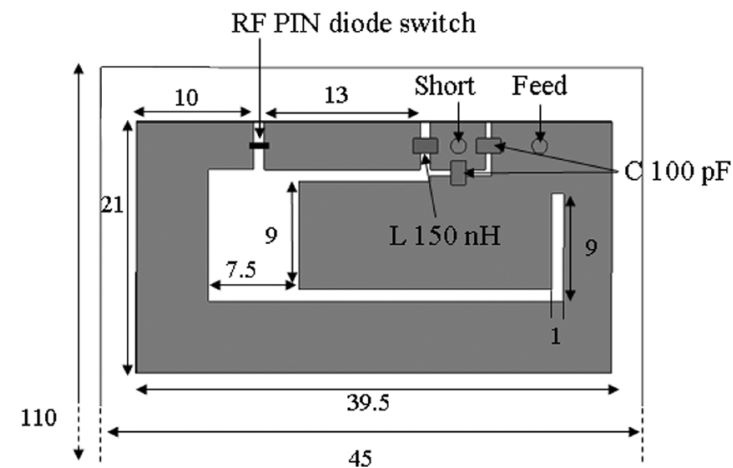

Fig. 1. Dual-band PIFA with an integrated tuning circuit. Dimensions are in $\mathrm{mm}$.

deformation of the radiation pattern and undesired resonances [18]. Furthermore, external tuning circuits increase the overall size of the antenna.

The tuning method presented in this paper is based on locating a RF switch directly in an antenna element. Unlike the situations proposed in [19], [20], no separate dc control circuit for the switch is used. The tuning circuit is integrated using reactive passive components that form separate paths for the RF signal and dc control voltage. The dc voltage is carried to the switch simultaneously with the RF signal. The proposed tuning method has been demonstrated with a single-feed dual-band PIFA operating in four distinct frequency bands. The antenna is tuned with a RF PIN diode switch and covers the frequency ranges appropriate to the GSM850, GSM900, GSM1800, PCS1900, and UMTS telecommunication standards. In order to investigate the effects of the tuning circuit on the antenna's radiation pattern and efficiency, a similar reference antenna, but without the tuning circuit, was also fabricated and tested.

Section II of this paper describes the new tuning method, and provides details of the new antenna structure. Following this in Section III the measured return losses, total efficiencies and radiation patterns of both the tunable antenna and the reference antenna are presented. Finally, discussions and conclusions are given in Sections IV and V.

\section{ANTENNA STRUCTURE}

Fig. 1 depicts the layout of a coaxial probe-fed dual-band PIFA. The structure incorporates an antenna element, feeding and short circuit pins, a RF diode switch, an inductor and two capacitors, as illustrated. The antenna is of a well-known PIFA type [17], [21], [22]. The rectangular patch is slit to form two separate paths for resonating currents, and this leads to dualband operation. The antenna element is built on a $0.8 \mathrm{~mm}$ thick $\mathrm{R} 4003 \mathrm{C}$ printed circuit board $\left(\varepsilon_{\mathrm{r}}=3.38, \tan \delta=0.0027\right.$ at 10 $\mathrm{GHz}$ ) with $35 \mu \mathrm{m}$ thick copper metallization. The antenna element has a $9.2 \mathrm{~mm}$ thick air substrate and it is placed on top of a $45 \mathrm{~mm} \times 110 \mathrm{~mm}$ reverse side grounded R4003C PCB. The geometrical dimensions and the values of the passive components were obtained by designing the antenna structure with the aid of commercial EM software, namely Ansoft HFSS V. 10.

The short circuit point is separated from the rest of the antenna element metallization by a narrow L-shaped slit. In order to generate a dc potential difference across the diode, two ca- pacitors $(\mathrm{C}=100 \mathrm{pF})$ and an inductor $(\mathrm{L}=150 \mathrm{nH})$ are connected in parallel with the slit. The capacitors, with low impedances at RF, act as a dc block and an RF short, and thus allow $\mathrm{RF}$ currents to flow freely in the antenna element. The inductor with high RF impedance blocks the RF signal and passes the dc. The dc voltage can be brought to the diode with the RF signal, so that there is a dc potential difference is between the feed and the short circuit pins. Consequently, changing the polarity of the dc voltage to produce either a forward bias (switch ON) or a reverse bias (switch OFF) condition for the diode will control the switch impedance.

The RF PIN diode is used here as a switch having either very high or low impedance depending on the applied dc bias. A comprehensive description of the principle of operation of the RF PIN diode switch and its use in frequency tuning of PIFAs has been presented by Louhos and Pankinaho in [10]. Furthermore, in the aforementioned paper a complete equivalent circuit for RF PIN diode switch, including the package parasitics, was presented. However, in the current work, simplified component models are used for describing the switch. In the forward biased state (ON state), the diode is assumed to act as an almost pure ohmic resistance and is modeled by a simple resistor. The reverse biased diode (OFF state) is assumed to act as a very small capacitance, which thus represents high impedance which in turn can regarded almost as an open circuit for RF frequencies. Use of different kinds of simplified diode models has previously reported [16], [23], [24] and they are able to predict the return losses of the tunable antenna with a reasonable accuracy, as will be shown later in this paper.

In the simulation model, the diode was modeled with a RLC boundary sheet with $2 \mathrm{ohm}$ resistance in the ON state and a 0.15 $\mathrm{pF}$ capacitance in the OFF state. These values are the same as those given for an Infineon BAR50-02V RF PIN diode switch. According to the manufacturer's data sheet, the diode switch has about $0.3 \mathrm{~dB}$ of insertion loss (with $10 \mathrm{~mA} \mathrm{dc}$ current) in the forward bias state and OFF-state isolation better than $20 \mathrm{~dB}$ over a frequency range up to $2 \mathrm{GHz}$ [25]. Values given in the datasheet are, however, referred to $50 \Omega$ impedance. The passive components were modeled with sheets having adequate capacitance and inductance values. The simulated return losses $\left(\mathrm{S}_{11}\right.$ in $\left.\mathrm{dB}\right)$ of the tunable antenna are presented in Fig. 2.

The simulations showed resonances at $890 \mathrm{MHz}$ and 1770 $\mathrm{MHz}$ while the switch was ON. The corresponding frequencies in the OFF state were 930 and $1960 \mathrm{MHz}$, respectively. The simultaneous frequency shift of both frequency bands can be explained by using the simulator to display the surface currents on the antenna, as shown in Fig. 3.

In the lower frequency band, actual switching occurs and a large amount of current flows through the diode in the ON state, and almost no current passes through the diode in the OFF state. Hence, frequency tuning of the lower band is simply based on modifying the length of the current path. The amount of current through the diode is smaller in the upper band than in the lower band. This indicates that the function of the switch is to load the antenna by changing the coupling between the two resonators separated by the narrow slit. When the switch is ON, strong surface currents occupy a larger area compared that for the OFF state, thus the resonant frequency is lower in the ON state. 


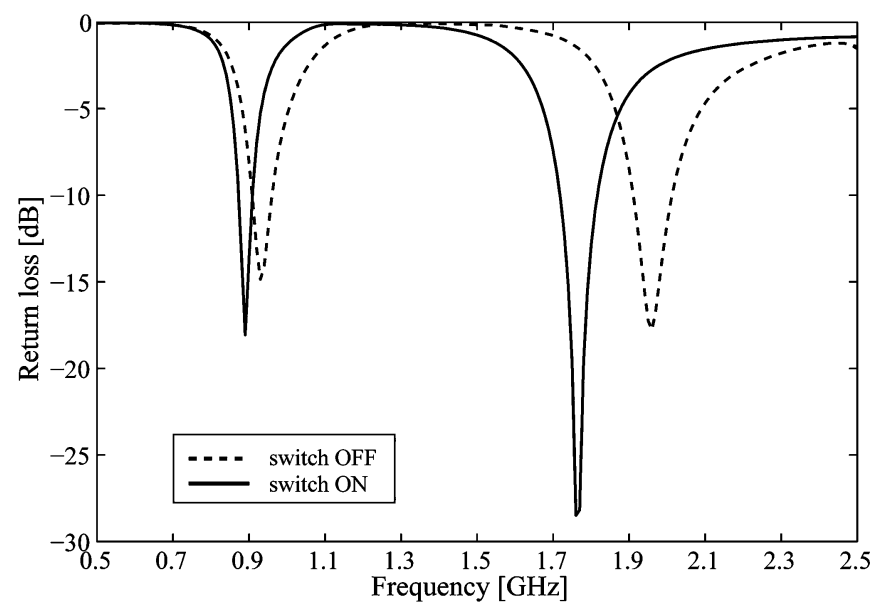

Fig. 2. Simulated return losses of the antenna with an integrated tuning circuit.

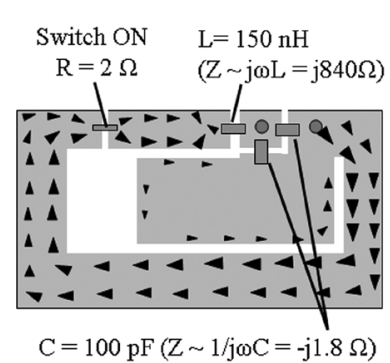

a)

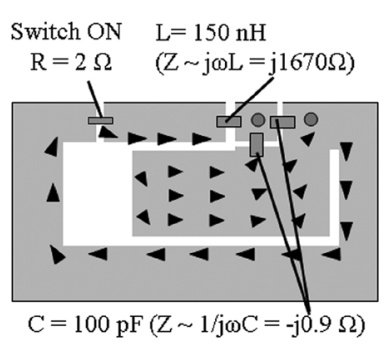

c)

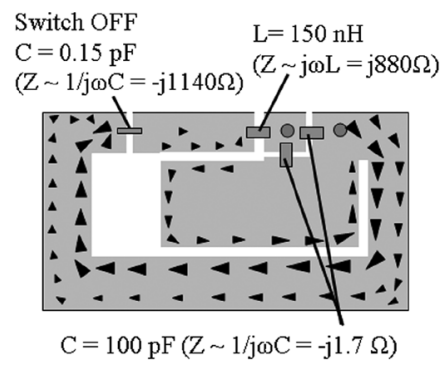

b)

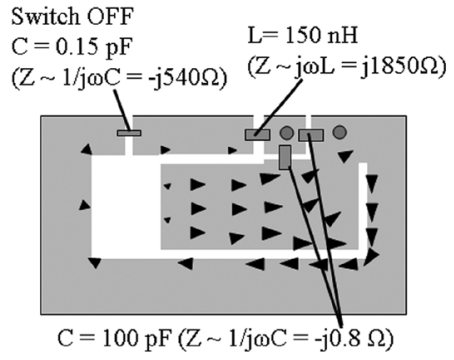

d)
Fig. 3. Simulated surface current distribution plots: (a) in the lower band when the switch is ON (890 MHz); (b) in the lower band when the switch is OFF (930 $\mathrm{MHz})$; (c) in the upper band when the switch is ON $(1770 \mathrm{MHz})$; (d) in the upper band when the switch is OFF (1960 MHz).

The PCBs for the antenna structures were fabricated using a standard photolithographic process. A BAR50-02V diode, along with standard 0805 passive components were soldered to the antenna element. The center conductor (diameter 1.3 $\mathrm{mm}$ ) of a standard $50 \Omega$ SMA through-connector was used as a feed pin and a round connector with the same diameter was used as a short-circuit pin. The reference antenna was similar to the tunable one, except that the diode, passive components and additional slits at the component positions were removed. Fig. 4 shows both of the fabricated antenna structures. The simulated return loss of the reference antenna is compared with that of the measured one in Section III.

\section{RESULTS}

For handset antennas, variations in the multipath environment and the varying orientation of the portable device, mean

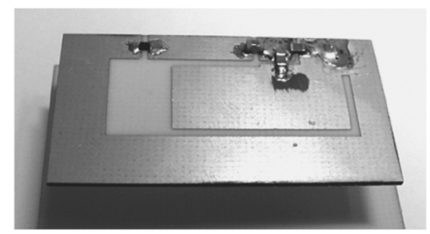

a)

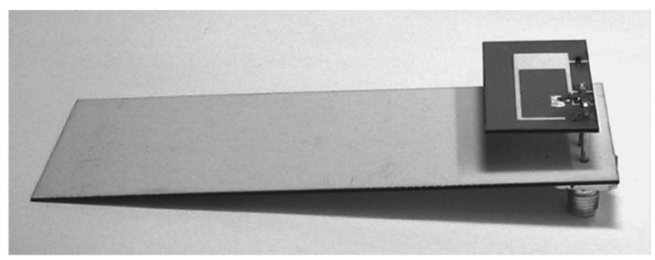

c)

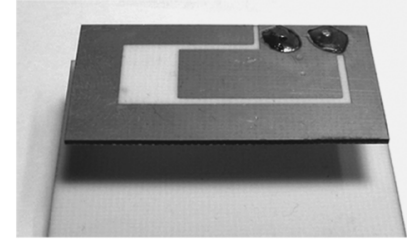

b)
Fig. 4. Fabricated antenna structures showing: (a) the antenna element of the PIFA with an integrated tuning circuit; (b) the antenna element of the reference PIFA without the tuning circuit; (c) the side view of the PIFA with an integrated tuning circuit.

that total efficiency or average gain are important figures-ofmerit. Furthermore, in tunable antennas efficiency deterioration caused by the tuning circuits, can be one of the major drawbacks. So as to address these issues, the electrical performance of the frequency tunable PIFA was characterized in terms of measured return loss, total efficiency, maximum radiation efficiency and far-field radiation patterns. The same measurements were also performed for the reference antenna.

\section{A. Return Loss}

A coaxial bias-T component was connected to a vector network analyzer that provided the dc control voltage for the switch during the return loss measurement. The ON and OFF conditions for the switch were obtained using $\pm 1 \mathrm{~V}$ (with a $20 \mathrm{~mA}$ forward current), respectively, to create the forward and reverse bias for the diode. The measured and simulated return losses of the tunable antenna are presented in Fig. 5. The simulation results are the same as in the Fig. 2.

When the switch is ON the resonances are at 854 and 1770 $\mathrm{MHz}$. The antenna covers the frequency ranges of the GSM850 (824-894 MHz) and GSM1800 (1710-1880 MHz) bands within at least $\mathrm{a}-3 \mathrm{~dB}$ impedance bandwidth. When the switch is OFF the resonant frequencies are 931 and $2025 \mathrm{MHz}$. The GSM900 (880-960 MHz) and UMTS (1920-2170 MHz) bands are thus covered. The PCS1900 (1850-1990 MHz) band is between the operating frequencies, so that the RX and TX portions are separated.

The simulated and measured return losses of the reference antenna are presented in Fig. 6. The antenna resonates at 815 and $1800 \mathrm{MHz}$. Compared with the tunable antenna in the ON state, slight upward frequency shifts of 40 and $30 \mathrm{MHz}$ were observed for the lower and upper resonant frequencies, respectively.

The agreement between the simulated and measured results was generally very good. However, there are small frequency shifts between the simulated and measured return losses of the tunable antenna. These frequency shifts are $-40 \mathrm{MHz}$ in the lower band in the switch ON-state and $+65 \mathrm{MHz}$ in the upper band in the switch OFF-state. However, the very good agreement 


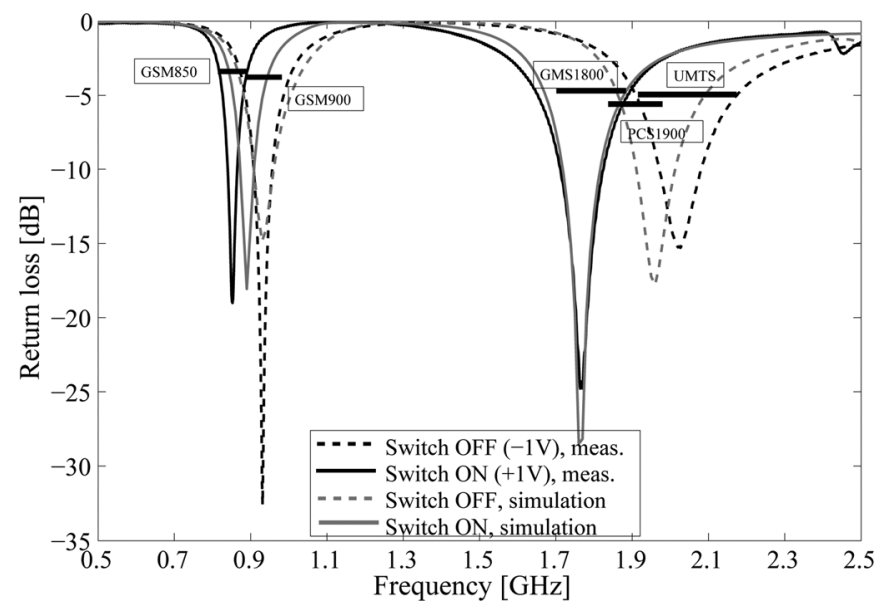

Fig. 5. Measured and simulated return losses of the antenna with an integrated tuning circuit.

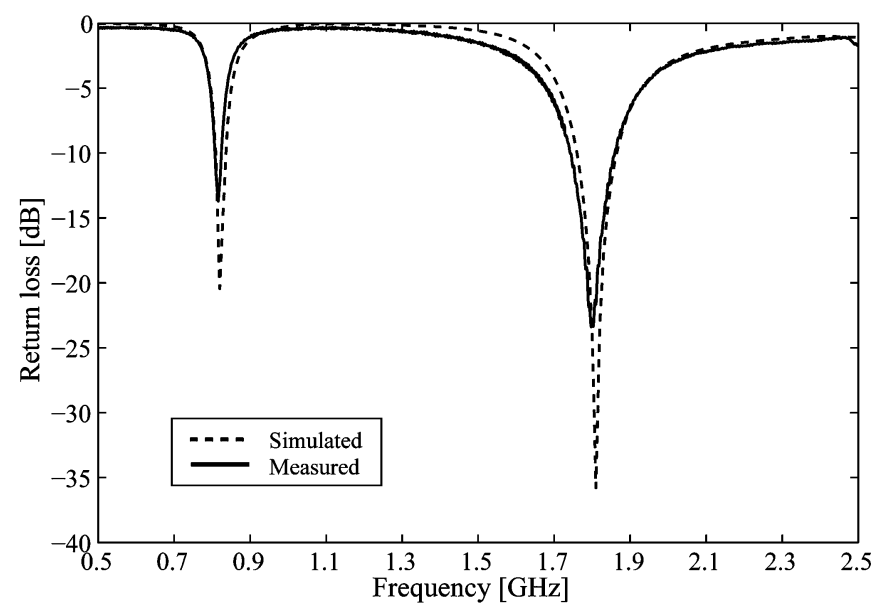

Fig. 6. Simulated and measured return loss of the reference antenna without the tuning circuit.

between the simulated and measured data for the reference antenna suggests that the frequency shifts observed for the tunable antenna can be attributed to the inaccuracy of the component models used, or possible fabrication and assembly errors. It should be noted that no account was taken of the parasitic components, such as lead inductance and packaging capacitance, associated with the PIN diode, and at the higher frequencies these parasitics could significantly affect the diode impedances in the ON and OFF states. It should be mentioned that it was found difficult to add an equivalent circuit consisting of several passive components to the EM simulation model.

\section{B. Efficiency}

A Satimo Starlab near-field chamber was used for the radiation pattern and total antenna efficiency measurements [26]. The frequency range of the measurement system started from 800 $\mathrm{MHz}$. The coaxial bias- $\mathrm{T}$ component was incorporated in the measurement system and the system was calibrated. The measured total efficiencies of the tunable antenna are presented in Fig. 7.

The results show peak values of $68 \%$ and $88 \%$ in the switched ON state and $65 \%$ and $85 \%$ in the OFF state. Thus the antenna

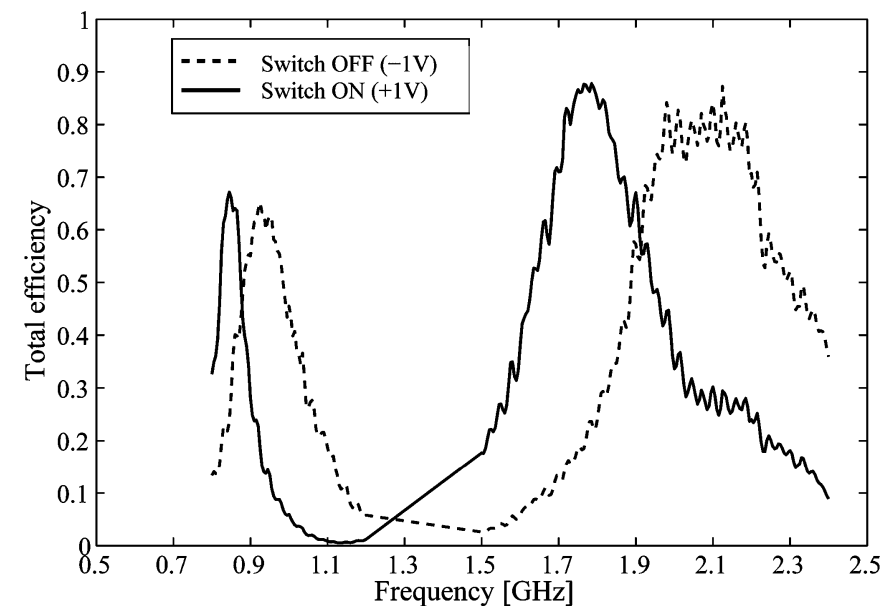

Fig. 7. Measured total efficiencies of the antenna with an integrated tuning circuit.

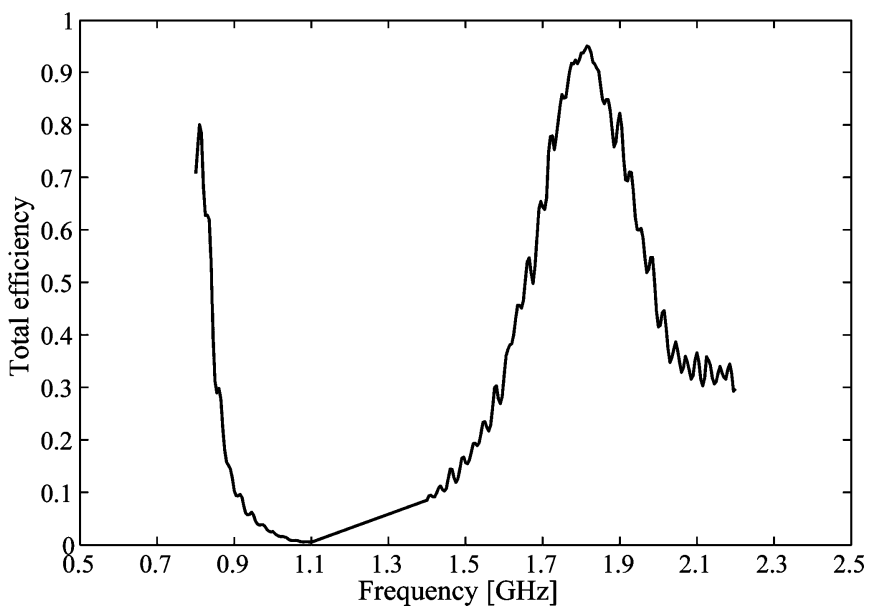

Fig. 8. Measured total efficiency of the reference antenna without the tuning circuit.

covers the frequency bands of GSM1800, PCS1900 and UMTS with a $65 \%$ total efficiency margin. The total efficiency coverage for the GSM900 and GSM850 bands were 50\% and 40\%, respectively.

It should be noted that besides the internal losses of the antenna structure, total efficiency also includes matching losses at the input port. The total efficiency $\left(\eta_{\text {tot }}\right)$ is defined as

$$
\eta_{\mathrm{tot}}=\eta_{\mathrm{rad}} *\left(1-|\Gamma|^{2}\right)
$$

where $\Gamma$ is the reflection coefficient at the input port, and $\eta_{\mathrm{rad}}$ is the radiation efficiency, which can be defined in terms of the radiated power $\left(\mathrm{P}_{\mathrm{rad}}\right)$ and the power lost in the antenna structure $\left(P_{\text {loss }}\right)$

$$
\eta_{\mathrm{rad}}=\mathrm{P}_{\mathrm{rad}} /\left(\mathrm{P}_{\mathrm{rad}}+\mathrm{P}_{\text {loss }}\right) .
$$

Here, the power loss can be further divided into losses due to the metallic antenna parts, dielectric losses and the loss caused by the tuning circuit components. The measured peak return loss values in Fig. 5 show that all the bands of the antenna exhibit 

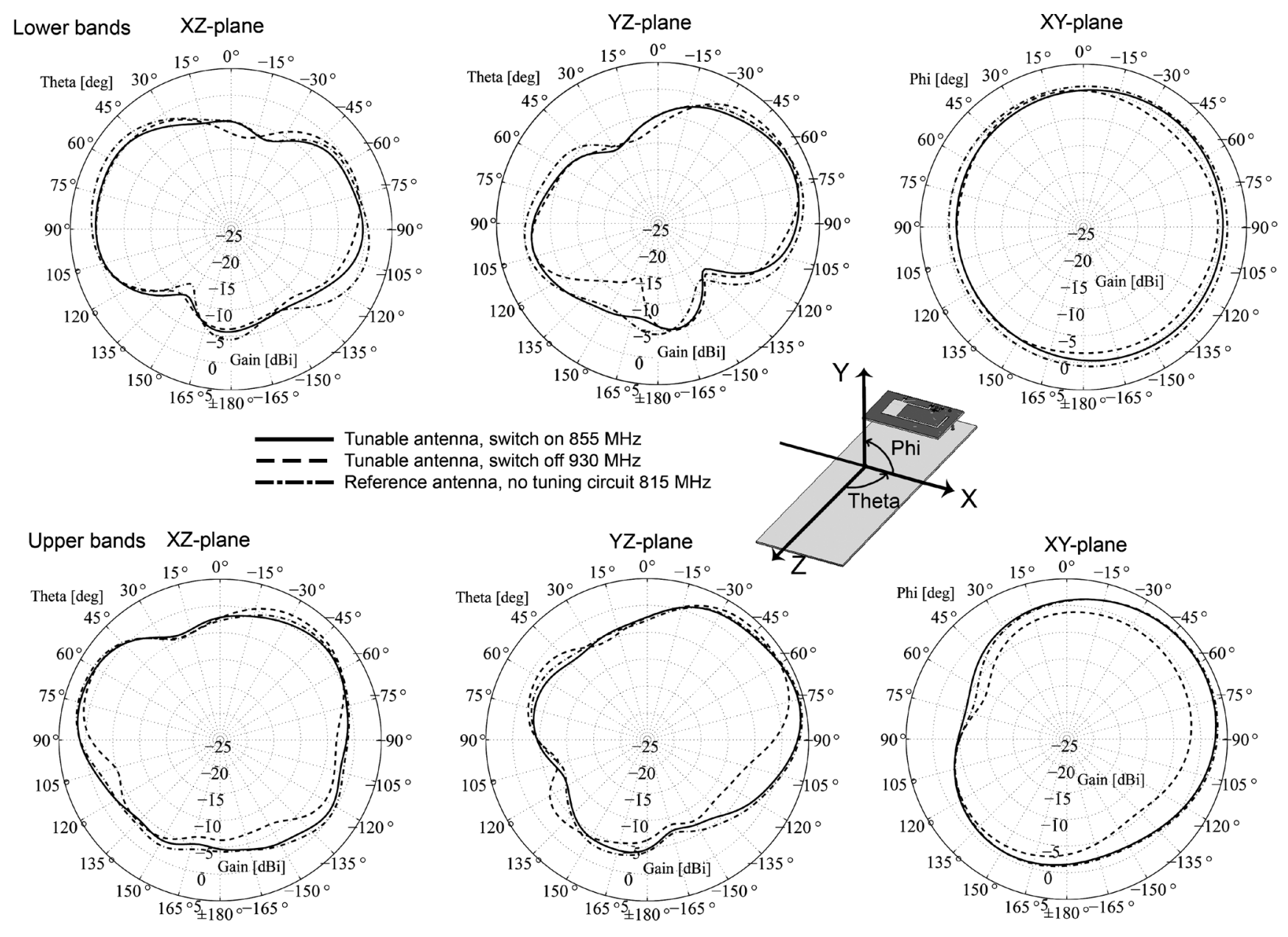

- Tunable antenna, switch on $1770 \mathrm{MHz}$

Tunable antenna, switch off $2025 \mathrm{MHz}$

Reference antenna, no tuning circuit $1800 \mathrm{MHz}$

Fig. 9. Measured far-field radiation patterns at the center operating frequencies of the tunable antenna and the reference antenna without the tuning circuit.

a good impedance match, with $\mathrm{S}_{11 \text { (peak) }}$ better than $-16 \mathrm{~dB}$. Thus the maximum total antenna efficiencies are nearly equal to the maximum radiation efficiencies, which are $69 \%$ and $88 \%$ in the switched ON state, and $65 \%$ and $87 \%$ in the OFF state.

The measured total efficiency of the reference antenna is presented in Fig. 8.

The result shows peak total efficiency values of $80 \%$ for the lower band and $95 \%$ for the upper band. Both antennas are built with low loss dielectrics, mainly air and partly R4003C PCB. In addition, highly conducting copper metallization was used. As a consequence, the dielectric and metallic losses were assumed to be small. Thus the reference antenna has high radiation efficiency, with peak values of $84 \%$ and $95 \%$. Finally, radiation efficiency deterioration caused by the tuning circuit can be estimate at less than $20 \%$ for the lower bands and less than $10 \%$ for the upper bands. No appreciable difference in the efficiencies between the switched ON state and the switched OFF state was observed.

\section{Radiation Pattern}

The measured far-field radiation patterns at the center operating frequencies of both antennas are presented in Fig. 9. The patterns of the lower frequency bands are near omni-directional and exhibit similarities with the pattern of a dipole antenna. The maximum lower band gain values of the tunable antenna are $1.8 \mathrm{dBi}$ in the ON state and $2.2 \mathrm{dBi}$ in the OFF state. The corresponding value for the reference antenna is $2.5 \mathrm{dBi}$. The antenna is able to maintain the shape of the patterns despite the presence and use of the tuning circuit. The patterns of the upper bands were found to be more directional, with maximum gains of 4 $\mathrm{dBi}$ (switch ON), $3.6 \mathrm{dBi}$ (switch OFF) and $4.3 \mathrm{dBi}$ (reference antenna). The upper band radiation patterns for the reference antenna and the switched ON tunable antenna are quite similar. The use of the tuning circuit, however, slightly tilts the pattern in OFF state. This can be seen best from the radiation plot in the $\mathrm{XY}$ plane, Fig. 9. Nevertheless, the antenna is able to maintain its efficiency and there is little degradation in the direction of radiation.

The essentially constant, dipole-like radiation patterns in the lower frequency bands can be attributed to the significant effect of ground plane radiation. It has been established in the literature that so-called chassis wave modes have a dominant radiation contribution in mobile phone antennas operating near the 900 $\mathrm{MHz}$ range [27]. At frequencies near $2 \mathrm{GHz}$, more radiation originates from the antenna element, and thus the antenna is more directional, and a slight tilt in the upper band patterns tends to occur when the antenna is tuned. 


\section{DISCUSSION}

The novel frequency tuning method presented in this paper has several beneficial features. The integrated tuning circuit allows greater independent design between the antenna and the rest of the device when compared with external tuning circuit techniques. This is because no parts of the tuning circuit, except for the bias-T for combining RF and dc, are situated outside the antenna element. Moreover, the method enables simultaneous tuning of several frequency bands, and the tuning circuit can be made of standard low-cost components. Another important feature related to the new method is its potential for maintaining high total antenna efficiency, both in terms of a good impedance match and high radiation efficiency.

In principle, the proposed concept can be extended to any kind of PIFA structure as well as other antenna types with a short circuit connection, such as IFAs and shorted monopole antennas. Replacing the RF diode with a very low loss RF MEMS switch may further increase the electrical performance of the antenna. In addition, $\mathrm{RF}$ varactors are potential candidates for providing continuous frequency tuning over the desired frequency band. Use of multiple switches or varactors in the same antenna element may also be considered to yield more versatile functions, such as greater sub-division of the overall frequency band, and also independent tuning of one or more sub-bands in a multiband antenna.

A valid performance comparison of different tuning methods for PIFAs is difficult, because a limited amount of efficiency data is available. Nevertheless, by using switchable tuning stubs, radiation efficiencies between $80-95 \%$ have been achieved [16], [17]. Also, significant 50-60\% drops in radiation efficiency, caused by the tuning circuits, have been reported for different tuning methods [10]. Essential system-level requirements, such as linearity and power handling capability of the active components, have to be considered when dealing with electrically tunable antennas. Ollikainen et al. in [16] presented a comprehensive study of single-band PIFAs that were tuned with switchable tuning stubs. The antennas operated near $900 \mathrm{MHz}$ and had tuning circuit components quite similar to the ones presented in this paper. It was shown that the antenna had very low distortion and it was capable of handling high power levels $(30 \mathrm{dBm})$.

For completeness, other important requirements such as switching time and dc power consumption, need to be considered. All of these issues are highly dependent on the system specifications and the properties of the selected active tuning circuit components. Whilst these aspects need further investigation, they do not detract from the essential merits of the proposed new technique.

\section{CONCLUSION}

A novel frequency tuning method for planar inverted-F antennas has been proposed, and validated through practical measurement. In this method, the tuning circuit is built into the antenna element by using discrete reactive passive components to form separate paths for the RF signal and the dc voltage, the latter being used to control the state of the active component. The dc voltage is applied to the antenna with the RF signal, and the tuning circuit is completely integrated. The effectiveness of the tuning method was demonstrated with a single-feed dual- band PIFA tuned with a RF PIN diode switch to operate in four frequency bands. The antenna covers the frequency ranges of the GSM1800, PCS1900 and UMTS telecommunication standards with a $65 \%$ total antenna efficiency margin. The total efficiency coverage of the GSM900 and GSM 850 bands are $50 \%$ and $40 \%$, respectively.

In order to investigate impact of the tuning circuit on the antenna's radiation pattern and efficiency, a reference antenna without the tuning circuit was fabricated and measured. It was shown that the tuning circuit reduces the radiation efficiency by less than $20 \%$ in the lower bands and less than $10 \%$ in the upper bands. The radiation patterns of the lower frequency bands remain essentially unchanged, despite the presence and use of the tuning circuit. However, in the upper band a slight tilt in the pattern occurs when the antenna is tuned.

The proposed frequency-tuning concept can be extended to more complex PIFA structures as well as other antenna types. Instead of the RF diode switch, RF MEMS components or RF varactors may used to further improve the electrical performance and functionality of the antennas.

\section{ACKNOWLEDGMENT}

The industrial partners of the TAMTAM technology project are acknowledged.

\section{REFERENCES}

[1] P. Lindberg, "Wide-band active and passive antenna solutions for hand held terminals," Ph.D. dissertation, Uppsala University, Sweden, Feb. 2007.

[2] R. F. Harrington, "Effect of antenna size on gain, bandwidth and efficiency," J. Res. Natl. Bur. Stand., vol. 64D, no. 1, pp. 1-12, Jan.-Feb. 1960.

[3] J. T. Aberle, S.-H. Oh, D. T. Auckland, and S. D. Rogers, "Reconfigurable antennas for portable wireless devices," IEEE Antennas Propag. Mag., vol. 45, no. 6, pp. 148-154, Dec. 2003.

[4] P. Sjöblom and H. Sjöland, "An adaptive impedance tuning CMOS circuit for ISM 2.4-GHz band," IEEE Trans. Circuits Syst. I, Reg. Papers, vol. 52, no. 6, pp. 1115-1124, Jun. 2005.

[5] F. Yang and Y. Rahmat-Samii, "Patch antennas with switchable slots (PASS) in wireless communications: Concepts, designs and applications," IEEE Antennas Propag. Mag., vol. 47, no. 2, pp. 13-29, Apr. 2005.

[6] C. G. Christodoulou, D. Anagnostou, and V. Zachou, "Reconfigurable multifunctional antennas," in Proc. IEEE Int. Workshop on Antenna Technology Small Antennas and Novel Metamaterials, Mar. 2006, pp. 176-179.

[7] J. Luo and M. Dillinger, "Reconfigurable radio technology demanding high end antenna solutions," in Proc. 12th Int. Conf. on Antennas and Propagation, Mar. 2003, vol. 1, pp. 378-384.

[8] H. Park, K. Chung, and J. Choi, "Design of a planar inverted-F antenna with very wide impedance bandwidth," IEEE Microw. Wireless Comp. Lett., vol. 16, no. 3, pp. 113-115, Mar. 2006.

[9] B. Sanz-Izquierdo, J. C. Batchelor, R. J. Langley, and M. I. Sobhy, "Single and double layer planar multiband PIFAs," IEEE Trans. Antennas Propag., vol. 54, no. 5, pp. 1416-1422, May 2006.

[10] J.-P. Louhos and I. Pankinaho, "Electrical tuning of integrated mobile phone antennas," in Proc. Antenna Applications Symp., Monticello, IL, 1999, pp. 69-97.

[11] L. M. Feldner, C. T. Rodenbeck, and C. G. Christodoulou, "Tunable electrically small UHF PIFA-as-a-package," in Proc. IEEE AP-S Int. Symp. Dig., Jun. 2006, pp. 185-188.

[12] N. C. Karmakar, P. Hendro, and L. S. Firmansyah, "Shorting strap tunable single feed dual band PIFA," IEEE Microw. Wireless Comp. Lett., vol. 13, no. 1, pp. 13-15, Jan. 2003.

[13] N. C. Karmakar, "Shorting strap tunable stacked patch PIFA," IEEE Trans. Antennas Propag., vol. 52, no. 11, pp. 2877-2883, Nov. 2004.

[14] P. K. Panayi, M. O. Al-Nuaimi, and I. P. Ivrissimtzis, "Tuning techniques for planar inverted-F antenna," IEEE Electron. Lett., vol. 37, no. 16, pp. 1003-1004, Aug. 2001. 
[15] H. Okabe and K. Takei, "Tunable antenna system for 1.9-GHz PCS handsets," in IEEE AP-S Int. Symp. Dig., 2001, vol. 1, pp. 166-169.

[16] J. Ollikainen, O. Kivekäs, and P. Vainikainen, "Low loss tuning circuits for frequency-tunable small resonant antennas," in Proc. 13th IEEE Int. Symp. on Personal, Indoor and Mobile Radio Communications (PIMRC), 2002, vol. 4, pp. 1882-1887.

[17] O. Kivekäs, J. Ollikainen, and P. Vainikainen, "Frequency-tunable internal antenna for mobile phones," in Proc. 12th Int. Symp. on Antennas (JINA), Nov. 2002, vol. 2, pp. 53-56.

[18] D. E. Anagnostou, G. Zheng, M. T. Chryssomallis, J. C. Lyke, G. E. Ponchak, J. Papapolymerou, and C. G. Christodoulou, "Design, fabrication, and measurements of an RF-MEMS-based self-similar reconfigurable antenna," IEEE Trans Antennas Propag., vol. 54, no. 2, pp. 422-432, Feb. 2006.

[19] P. Panaia, C. Luxey, G. Jacquemod, R. Staraj, G. Kossiavas, L. Dussopt, F. Vacherand, and C. Billard, "MEMS-based reconfigurable antennas," in IEEE Int. Symp. on Industrial Electronics, 2004, vol. 1, pp. 175-179.

[20] N. C. Karmakar, "Biasing considerations of a switched parasitic planar inverted-F antenna," in IEEE AP-S Int. Symp. Dig., Jul. 2005, vol. 4b, pp. 60-63.

[21] S. Tarvas and A. Isohätälä, "An internal dual-band mobile phone antenna," in IEEE AP-S Int. Symp. Dig., Jul. 2001, vol. 1, pp. 266-269.

[22] P. Lindberg and E. Öjefors, "A bandwidth enhancement technique for mobile handset antennas using wave traps," IEEE Trans. Antennas Propag., vol. 54, no. 8, pp. 2226-2233, Aug. 2006.

[23] S. Nikolaou, R. Bairavasubramanian, C. Lugo, Jr, I. Carrasquillo, D. C. Thompson, G. E. Ponchak, J. Papapolymerou, and M. M. Tentzeris, "Pattern and frequency reconfigurable annular slot antenna using PIN diodes," IEEE Trans. Antennas Propag., vol. 54, no. 2, pp. 439-448, Feb. 2006.

[24] H. Aissat, L. Cirio, M. Grzeskowiak, J.-M. Laheurte, and O. Picon, "Reconfigurable circularly polarized antenna for short-range communication systems," IEEE Trans. Microw. Theory Tech., vol. 54, no. 6, pp. 2856-2863, Jun. 2006.

[25] Datasheet for BAR50-02V RF PIN-Diode Switch Infineon Technologies [Online]. Available: http://www.infineon.com/upload/Document/ cmc_upload/documents/081/844/bar50series_1.pdf

[26] P. O. Iversen, P. Garreau, and D. Burrel, "Real-time spherical near-field handset antenna measurements," IEEE Antennas Propag. Mag., vol. 43, pp. 90-94, Jun. 2001.

[27] P. Vainikainen, J. Ollikainen, O. Kivekäs, and I. Kelander, "Resonator-based analysis of the combination of mobile handset antenna and chassis," IEEE Trans. Antennas Propag., vol. 50, no. 10, pp. 1433-1444, Oct. 2002.

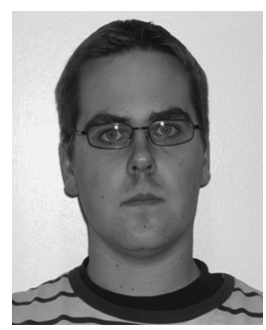

Mikko Komulainen (S'05) received the M.Sc. degree in electronics from the University of Oulu, Oulu, Finland, in 2005, where he is currently working toward the Doctorate degree.

His research interests include tunable RF and microwave components and functional microwave materials.

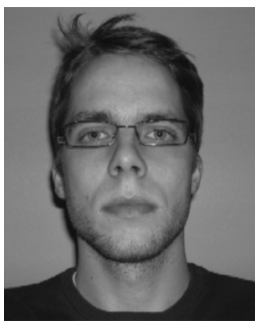

Markus Berg (S'05) received the M.Sc. degree in electrical engineering from the University of Oulu, Oulu, Finland, in 2005, where he is currently working toward the Doctorate degree.

Since 2005 he has worked as a Researcher at the Telecommunication laboratory of the University of Oulu. His research interests include small antennas for mobile terminals.

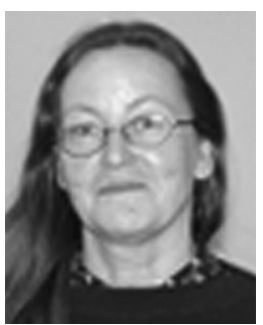

Heli Jantunen (M'02) was born in Oulu, Finland, in 1958. She received the M.Sc. degree in astronomy and physics, the Diploma in education, the M.Sc. degree in electrical engineering, and the Dr. Tech. degree in electrical engineering (with honors) all from the University of Oulu, in 1982, 1985, 1989, and 2001, respectively.

After spending 10 years in the industry, she joined the Microelectronics and Materials Physics Laboratories, University of Oulu, in 1999, where she is currently a Full Professor. Since 2004, she has been also the Head of Electronics Materials, Packaging and Reliability Techniques (EMPART) Research Group of Infotech Oulu, which gathers together six research groups with versatile background. The research of the group focuses on development of novel electronics, RF and photonics applications by implementation of advanced microwave materials, smart structures and nanotechnology into multifunctional micromodules. She has been or is currently engaged in research projects supported nationally (Finnish Funding Agency of Technology and Innovation, Academy of Finland) and internationally (Nordic Innovation Centre, EU). She holds several national and international patents.

Prof. Jantunen has been a member of the American Ceramic Society since 2002, and is also a Steering Committee Member of research and technology programs of the Academy of Finland and the Finnish Funding Agency of Technology and Innovation. She has been an Invited Speaker, Session Chair, and Scientific Committee Member in several international scientific conferences, General Chair of the International Conference of Microwave Materials and their Applications in 2006, and a Reviewer for scientific journals.

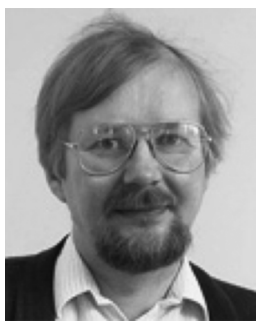

Erkki T. Salonen received the M.Sc., Licentiete of Science, and Doctor of Science in Technology degrees from Helsinki University of Technology (TKK), Espoo, Finland, in 1979, 1986, and 1993, respectively.

From 1984 to 1996, he was in charge of the radio wave propagation studies at the Radio Laboratory, Helsinki University of Technology. Since 1997, he has been a Professor of radio engineering in the Telecommunication Laboratory, University of Oulu. His main research interests include antennas and propagation in radio communications.

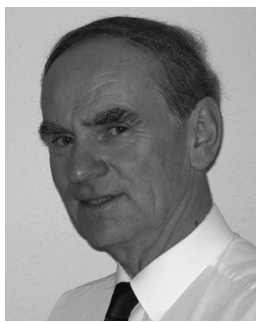

Charles Free (M'99) worked as a Research Engineer in industry before moving to academia. Until 2001, he was a Reader in Computer Communications at Middlesex University, London, U.K. He is now a Reader in Microwave Technology at the University of Surrey, Surrey, U.K., where his research interests are primarily in millimeter-wave planar circuits and antennas. He is the author of over 100 publications. 